\title{
Gene mutations and three-dimensional structural analysis in 13 families with severe factor X deficiency
}

\author{
Flora Peyvandi, ${ }^{1}$ Marzia Menegatti, ${ }^{1}$ Elena Santagostino, ${ }^{1}$ Sepideh Akhavan, ${ }^{1}$ James Uprichard, ${ }^{2}$ \\ David J. Perry, ${ }^{2}$ Stephen J. Perkins ${ }^{3}$ and Pier M. Mannucci ${ }^{1}{ }^{1}$ Angelo Bianchi Bonomi Haemophilia and \\ Thrombosis Centre and Fondazione Luigi Villa, IRCCS Maggiore Hospital and University of Milan, Italy, ${ }^{2}$ Haemophilia \\ Centre and Haemostasis Unit, Department of Haematology, and ${ }^{3}$ Department of Biochemistry and Molecular Biology, \\ Royal Free and University College Medical School, Royal Free Campus, London, UK
}

Received 26 September 2001; accepted for publication 17 December 2001

\begin{abstract}
Summary. Factor $\mathrm{X}(\mathrm{FX})$ deficiency is a rare autosomal recessive disorder. The phenotype and genotype of 15 Iranian patients with FX deficiency from 13 unrelated families with a high frequency of consanguinity were analysed. Five different assays identified four patients from three families with a discrepancy between low-FX coagulant activity (FX:C) and higher-FX antigen (FX:Ag) (a type II deficiency). The remaining 11 patients had parallel reductions of FX:C and FX:Ag (a type I deficiency). Nine different homozygous candidate mutations were identified, of which eight were novel. The four type II cases were associated with an $\operatorname{Arg}(-1) \mathrm{Thr}$ missense mutation in the prepropeptide: $\operatorname{Arg}(-1)$ is highly conserved in all vitamin K-dependent proteins. Four type I mutations (Gly78Asp, Cys81Tyr,
\end{abstract}

Gly94Arg and Asp95Glu) were localized to the EGF-1 and EGF-2 domains, for which molecular views showed that the protein folding would be disrupted. The type I mutation Gly222Asp was localized in the catalytic domain of FX, and is sufficiently close to the Asp-His-Ser catalytic triad to disrupt its correct protein folding. The two type I splice site mutations were IVS1+3, A $\rightarrow$ T and IVS2-3, T $\rightarrow$ G. These novel homozygous FX mutations were consistent with their phenotypes and agree with experimental data from knockout mice, indicating that FX is an essential protein for survival.

Keywords: FX mutations, FX deficiency, FX deficiency phenotype analysis.
Factor X (FX), the zymogen of the serine protease FXa, occupies a central position in the coagulation cascade, and represents the point of convergence between the intrinsic and extrinsic pathway of blood coagulation. FX is synthesized by the liver and circulates in plasma at a concentration of $8-10 \mu \mathrm{g}$ as a two-chain protein with a $17-\mathrm{kDa}$ light chain disulphide linked to a 45-kDa heavy chain. The light chain contains a $\gamma$-carboxyglutamic acid (Gla) domain necessary for a $\mathrm{Ca}^{2+}$-dependent conformational change associated with phospholipid binding and two epidermal growth factor (EGF) domains. The heavy chain contains the catalytic serine protease domain, which is structurally homologous to that of other coagulation serine proteases. Once activated, FX associates with its cofactor factor Va on a membrane surface to form the prothrombinase complex.

The FX gene (F1O) contains eight exons and is located on chromosome 13q34 (Tuddenham \& Cooper, 1994). Each of

Correspondence: Dr Flora Peyvandi, Angelo Bianchi Bonomi Haemophilia and Thrombosis Centre, Via Pace, 9-20122, Milan, Italy. E-mail: flora.peyvandi@unimi.it the exons of $F 10$ encodes a specific protein domain (Furie \& Furie, 1988). Exon I encodes the signal peptide, exon II the propeptide and Gla domain, and exon III a short linking segment of aromatic amino acids. Exons IV and V encode the two EGF domains, exon VI encodes the activation peptide at the N-terminus of the heavy chain, and exons VII and VIII encode the serine protease domain with the catalytic triad His236, Asp228 and Ser379. FX is synthesized with a 40-residue prepropeptide, which contains the hydrophobic signal sequence (residues -37 to -22 ) that directs the secretion of FX (Leytus et al, 1986). The propeptide appears to be important in directing intracellular FX and for post-translational modification (Leytus et al, 1986).

FX deficiency is a rare haemorrhagic condition, inherited as an autosomal recessive trait. Patients with severe FX deficiency are the most seriously affected among patients with rare coagulation defects (Peyvandi \& Mannucci, 1999). The more frequent symptoms in FX-deficient patients are haemarthroses, muscle haematomas, umbilical cord bleeding, and gastrointestinal and central nervous system (CNS) bleeds (Peyvandi et al, 1998). To date, 39 
missense mutations and seven deletions in the FX gene have been reported (see Cooper et al, 1997; Perry, 1997; Miyata et al, 1998a,b; Nobauer-Huhmann et al, 1998; Zama et al, 1999; Bereczky et al, 2000; Millar et al, 2000; Deam et al, 2001; Iijima et al, 2001; Simioni et al, 2001, and references therein). To extend the knowledge on naturally occurring mutations that cause FX deficiency, 15 patients from 13 unrelated Iranian families with severe FX deficiency have been examined using molecular analysis and three-dimensional structure analysis. The great majority of these patients were born from first cousin consanguineous marriages. Therefore, in contrast with the majority of previously reported studies, this study reports data from homozygous patients, enabling us to define the clinical phenotype associated with the presence of one abnormal allele without interference or compensation by the other allele. Further to previously described mutations, most of which have been located within the catalytic serine protease domain, we describe the effect of four novel mutations in the two EGF domains and one in the serine protease domain.

\section{PATIENTS AND METHODS}

Patients. Fifteen FX-deficient patients from 13 unrelated families (12 men and 4 women, aged 2-55 years) were studied. All families were of Iranian origin. Eleven out of the 15 patients were the offspring of marriages between first cousins. Informed consent for investigation was obtained from all individuals. To establish the type and severity of bleeding symptoms, we used a questionnaire that was specially designed to collect focused and objective information from patients (Lak et al, 2000). Severe bleeders were classified as those who had spontaneous and/or life-threatening bleeding episodes, such as haemarthroses, muscle haematomas, and umbilical cord, gastrointestinal and CNS haemorrhages. Mild bleeders were those who bled only after trauma or surgery or who had bleeding problems such as epistaxis or menorrhagia. Non-bleeders were those with no history of bleeding.

Factor $X$ assays. Five different assays were used on samples from patients and their related family members. The FX coagulation activity (FX:C) assay methods involved a one-stage prothrombin time (PT) and an activated partial thromboplastin time (APTT) bioassay, both performed on an ACL3000 automated analyser (Instrumentation Laboratory, Milan, Italy), a manual onestage Russell viper venom (RVV) assay (Diagen, Thame, UK), and a two-stage chromogenic assay (Chromogenics, Instrumentation Laboratory, Milan, Italy). The FX:C values were calculated using, as a reference, plasma pooled from 40 normal individuals $(20 \mathrm{men}$, and 20 women who were not pregnant and who were not taking oral contraceptives). The reference plasma was assigned an arbitrary FX:C-value of 100\%. In Iran, FX:C was assayed with a PT assay method using rabbit thromboplastin and FX:C-deficient plasma from patients with severe deficiency and no FX:C inhibitor. Levels of FX antigen (FX:Ag) were measured using an in-house enzyme immunoassay. Plastic 96-well microtitre plates were coated overnight at room temperature with rabbit anti-human FX polyclonal antibody (Dako, Ely, UK) diluted 1:800. After removal of the coating solution with a washing buffer, a standard curve was constructed with serial dilutions of pooled normal plasma in the buffer, and samples were incubated for $2 \mathrm{~h}$ at room temperature. The plates were then washed and reincubated with a 1:650 dilution of horseradish peroxidase conjugated rabbit antihuman FX polyclonal antibody used in the coating step for $2 \mathrm{~h}$ at room temperature. The enzymatic activity was detected by ortho-phenylene-diamine; the reaction was stopped with a solution of $\mathrm{H}_{2} \mathrm{SO}_{4}$ and optical density was determined at $492 \mathrm{~nm}$. Results from this in-house enzyme immunoassay were compared with those from a commercial enzyme immunoassay kit (Dako, Ely, UK) using the same coating and labelling antibody and found to give similar FX:Ag values in each patient plasma tested. FX:Ag levels were calculated using as a reference the same pooled plasma used for the FX:C assays. Sensitivity of the assay was $0 \cdot 1 \%$ of normal FX:Ag levels (i.e. $100 \mathrm{ng} / \mathrm{ml}$ ), the intra-assay coefficient of variation was $5 \%$ and the interassay coefficient of variation, $8 \%$.

DNA analysis. Genomic DNA was extracted from peripheral blood leucocytes according to standard protocols. Following DNA extraction, the coding region, intron/exon boundaries and the $5^{\prime}$ and $3^{\prime}$ UTR of the FX gene were amplified by polymerase chain reaction (PCR) and screened for mutations by single strand conformation polymorphism analysis (SSCP) (Orita et al, 1989). Direct sequencing of amplified DNA fragments was used to confirm and identify the presence of any mutations.

Polymerase chain reaction. Amplification reactions were carried out in 30- $\mu \mathrm{l}$ volumes and comprised $100 \mathrm{ng}$ of DNA, 10 pmoles of each oligonucleotide primer, $240 \mu \mathrm{mol} / \mathrm{l}$ of each dNTP, $1.5 \mathrm{mmol} / \mathrm{l} \mathrm{MgCl}_{2}$, $50 \mathrm{mmol} / \mathrm{l} \mathrm{KCl}, 10 \mathrm{mmol} / \mathrm{l}$ Tris-HCl pH 8.3 and 2 units of Taq polymerase (PE, Applied Biosystems, UK). Reactions were denatured at $95^{\circ} \mathrm{C}$ for $5 \mathrm{~min}$, and then 35-40 cycles of amplification were performed. On the last cycle, the extension time was increased to $10 \mathrm{~min}$. The primer sequences and amplification parameters are available on request.

Single strand conformation polymorphism (SSCP) analysis. In total, $10 \mu \mathrm{l}$ of the final PCR product was mixed with $25 \mu \mathrm{l}$ of denaturation buffer $(95 \%$ formamide, $0.05 \%$ Bromophenol blue, $10 \mathrm{mmol} / \mathrm{l} \mathrm{NaOH})$, denatured for $10 \mathrm{~min}$ at $94^{\circ} \mathrm{C}$, then placed on ice for $2 \mathrm{~min}$. Then, $20 \mu \mathrm{l}$ of each denatured sample was loaded onto a $10 \%$ polyacrylamide gel (acrylamide:bisacrylamide) in $1 \times$ Trisborate-EDTA buffer $(0.09 \mathrm{mmol} / \mathrm{l}$ Tris-borate, $2 \mathrm{mmol} / \mathrm{l}$ EDTA). Gels were pre-run at $150 \mathrm{~V}$ at $15^{\circ} \mathrm{C}$ for $45 \mathrm{~min}$ in $1 \times$ Tris-borate-EDTA buffer before loading, then electrophoresis was carried out at $250 \mathrm{~V}$ for $5-6 \mathrm{~h}$ at $15^{\circ} \mathrm{C}$ (fragment size $\approx 250-300 \mathrm{bp}$ ). DNA fragments were visualized by silver staining (Promega, Milan, Italy). The ratios of acrylamide:bisacrylamide were different for the different FX exons and information is available on request. Supplementation of the gels with urea was used only in the study of exon 7 and the promoter. 
Sequence analysis. PCR products were purified (Qiagen Purification Kit, Milan, Italy) and directly sequenced using an ABI Prism310 genetic analyser automated sequencer (PE Applied Biosystems, Milan, Italy) with the same primers used for the amplification reactions, except for exon 1, which required a different sequencing primer. Any mutation identified by direct sequencing was confirmed by repeat sequencing and restriction enzyme digestion if associated with the creation or loss of a specific restriction enzyme site.

To confirm that our genetic variants were not frequent polymorphisms, all mutations were also investigated in 200 alleles in an Iranian control population.

Structural analysis of FX mutations. The crystal coordinates of FX were obtained from the Protein Data Bank (codes 1ezq, 1fOr, 1fOs, 1fax, 1hcg, 1xka, 1xkb) (Padmanabhan et al, 1993; Brandstetter et al, 1996; Kamata et al, 1998; Maignan et al, 2000). Protein structures were visualized using the INSIGHT II 98.0 program package (Biosym/MSI, San Diego, CA, USA) on Silicon Graphics INDY workstations, using Crystal Eyes stereo glasses for detailed inspections. The quantitative assignment of the experimentally observed main-chain secondary structure of each protein was made using the DSSP (Definition of Secondary Structure of Proteins) programme (Kabsch \& Sander, 1983); Fig 1 shows the $\alpha$-helix and $\beta$-strand contents (denoted $\mathrm{H}$ and $\mathrm{E}$ respectively) and other types of secondary structures including loops and turns (T, S, B and G). The quantitative
Human
Mutation
Mutation
DSSP

COMPARER

Human

Mutation

Mutation

DSSP STRUCTURE COMPARER

Human

Mutation

Mutation

DSSP

STRUCTURE

COMPARER

Human

Mutation

DSSP

STRUCTURE

COMPARER

Human

Mutation

DSSP

STRUCTURE

COMPARER

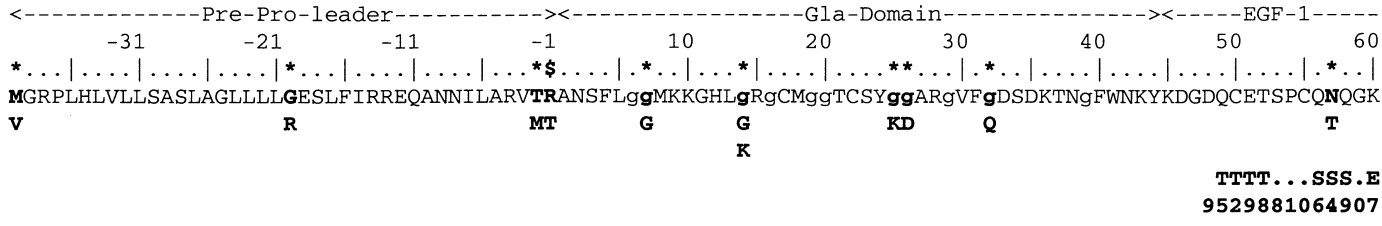

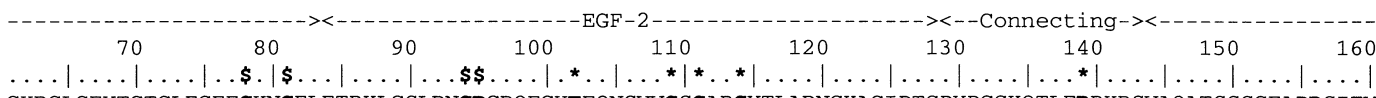
CKDGLGEYTCTCLEGFEGKNCELFTRKLCSLDNGDCDQFCHEEQNSVVCSCARGYTLADNGKACIPTGPYPCGKQTLERRKRSVAQATSSSGEAPDSITW

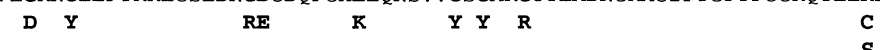

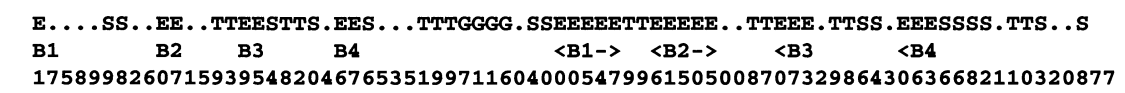

Fig 1. Protein sequence and structure analysis of human Factor X. The 39 previously known mutations are identified by asterisks (*); six of the eight novel mutations in this study are identified by dollar symbols (\$), and the three catalytic triad residues are identified by hatches (\#). The Gla, EGF and catalytic serine protease domain assignments are shown above the sequence numbering for FX (SWISSPROT code P00742). In the Gla domain, Gla residues are identified by ' $\mathrm{g}$ '. The DSSP output shows the consensus secondary structures from seven crystal structures of FX when these correspond to experimentally observed structures. No output is shown if no structures were present. The $\beta$-strands in the two EGF domains (denoted by E in the DSSP output) are each labelled B1-B4, and those in the serine protease subdomains are labelled A-O. The $\alpha$-helices (denoted by $\mathrm{H}$ ) in the serine protease subdomains are labelled A1-A3. The comPARER output shows a value (0-9), which represents the consensus solvent accessibility of each side-chain in FX. Exposed residues are identified by values of 2-9, and buried residues by values of 0 or 1 . 
assignment of solvent-exposed residue side-chains was made using the COMPARER programme by the Lee and Richards method of using a water molecule radius of 1.4 А (Lee \& Richards, 1971; Sali \& Blundell, 1990). Side-chains were defined as solvent-exposed if their accessibilities ranged between $20 \%$ and $100 \%$ (values 2-9 in Fig 1) and buried if these ranged between $0 \%$ and $19 \%$ (values of 0 and 1 in Fig 1 ).

Splice-site computer analysis. A computer-assisted analysis of splice-sites sequences and prediction for these sequences utilized the Berkley Drosophila Genome Project human database website (http://www.fruitfly.org/seq_tools/ spliceAbst.html).

\section{RESULTS}

\section{Phenotype analysis}

Table I summarizes the FX assays for each of 15 patients from the 13 unrelated families and the severity of their bleeding symptoms. In 11 out of 15 patients, low levels of both FX:C by all four methods and FX:Ag were observed, and these were assigned as type I deficiencies. In the remaining four patients (B1 and B2 from the same kindred; $\mathrm{C}$ and D from unrelated kindred), low levels of FX:C were observed in contrast to the normal levels of FX:Ag, and these were assigned as type II deficiencies (i.e. the presence of a dysfunctional FX molecule). Only patient $\mathrm{M}$ had FX levels below the assay sensitivity (1\%) using all assays for FX:C and FX:Ag.

The results obtained by the five assays showed marked variation in the sensitivity of the chromogenic assay compared with the three coagulation assays for individual samples. The pattern across the five methods was consistent within family members (data not shown). In 9 out of 15 patients, the FX:C result at diagnosis (in the referring Iranian hospital) was confirmed by our subsequent testing. Two patients ( $\mathrm{H} 1$ and $\mathrm{H} 2$ ) had a higher level of coagulant activity measured by the RVV test compared with PT, APTT, chromogenic and FX:Ag levels. In six patients (D, F, G, H1 and I) with severe bleeding symptoms (umbilical cord bleeding, recurrent haematomas and haemarthroses, haematuria, gastrointestinal bleeding), there was a discrepancy between the FX assay results at diagnosis in Iran and the subsequent value obtained in our laboratory. This discrepancy is probably due to traces of exogenous FX introduced by replacement therapy, which is frequently needed in these severely affected patients (Peyvandi et al, 1998). Four out of 15 severely affected patients (B1, B2, C and D) had normal borderline chromogenic activity and antigen levels in contrast to their low PT, APTT and RVV coagulation assays, indicating a type II deficiency. Eight samples from severely affected patients (E, F, G, H1, H2, I, J and K) had measurable but reduced chromogenic activity, and the patients would have been misdiagnosed if the chromogenic assay had been used alone. In two patients (A and L) only two out of the five FX assays (APTT and FX:Ag) were carried out because of limited amounts of available plasma.

\section{Novel mutations}

Six of the eight previously unreported mutations were missense mutations and two were splice-site mutations, all of which were homozygous.

The mutation $\operatorname{Arg}(-1)$ Thr present in exon 2 encoding the prepropeptide was present in four patients from three unrelated families (kindred B, C and D) who had low FX:C levels associated with normal borderline levels of FX:Ag and chromogenic assays (type II deficiency). A small discrepancy between PT, APTT and RVV values of patients B1, B2 and $\mathrm{C}$, D was observed. This discrepancy could be the result of small traces of the last substitutive treatment before sample collection. These patients need frequent treatment because they suffer from recurrent haemarthroses, haematomas and umbilical cord bleeding.

Two novel mutations, Gly78Asp and Cys81Tyr, present in exon 4 encoding EGF-1, were detected in three families with type I deficiencies (E, F and G). Patient E with Gly78Asp mutation presented with severe bleeding after circumcision, recurrent haemarthroses, haematomas, haematuria and mucosal bleeding. This patient required frequent replacement therapy with fresh frozen plasma, factor IX complex concentrates and red cells.

Two associated novel mutations Gly94Arg and Asp95Glu, present in exon 5 and encoding the EGF-2 domain, were identified in two siblings ( $\mathrm{H} 1$ and $\mathrm{H} 2$ ) who were not born from a consanguineous marriage. Both $\mathrm{H} 1$ and $\mathrm{H} 2$ were homozygous for these two mutations and both patients presented with recurrent haematomas, haemarthroses, epistaxes and oral bleeding. Both patients came from a small village of an Iranian Turkish ethnic group.

The last novel missense mutation (Gly222Asp), found in two apparently unrelated kindred ( $\mathrm{J}$ and $\mathrm{K}$ ), was in exon 7 encoding the catalytic domain of FX and was associated with a type I phenotype. Patient J has recurrent haematomas and haemarthroses, haematuria and epistaxis. Patient $\mathrm{K}$, with the same mutation, presented with epistaxis and gastrointestinal bleeding that required several blood transfusions.

The first splice-site mutation was localized at nucleotide +73 (+1 denotes the first nucleotide of the ATG start codon, numbering in accordance with GenBank, accession no. 12738260, 2001). In patient $A$, a homozygous $A \rightarrow T$ transition within intron A (IVS1+3) was associated with a parallel reduction in both FX:Ag and FX:C (APTT). The 8 -year-old boy with this mutation suffered from umbilical cord bleeding, haematoma, epistaxis and CNS bleeding. The second splice-site mutation was localized at nucleotide +15599 . Patient L showed a homozygous $\mathrm{T} \rightarrow \mathrm{G}$ transversion within intron B (IVS2-3), associated with type I deficiency. This patient suffered from recurrent knee haemarthroses, haematomas, epistaxes and oral bleeding.

\section{Previously reported mutation}

The mutation Gly204Arg in exon 7, identified in patient I, had been reported previously in a one-year-old boy, whose ethnic group was not given, with severe bleeding history and reduced FX:C and FX:Ag (Bereczky et al, 2000). Earlier molecular modelling suggested that the mutant protein is 


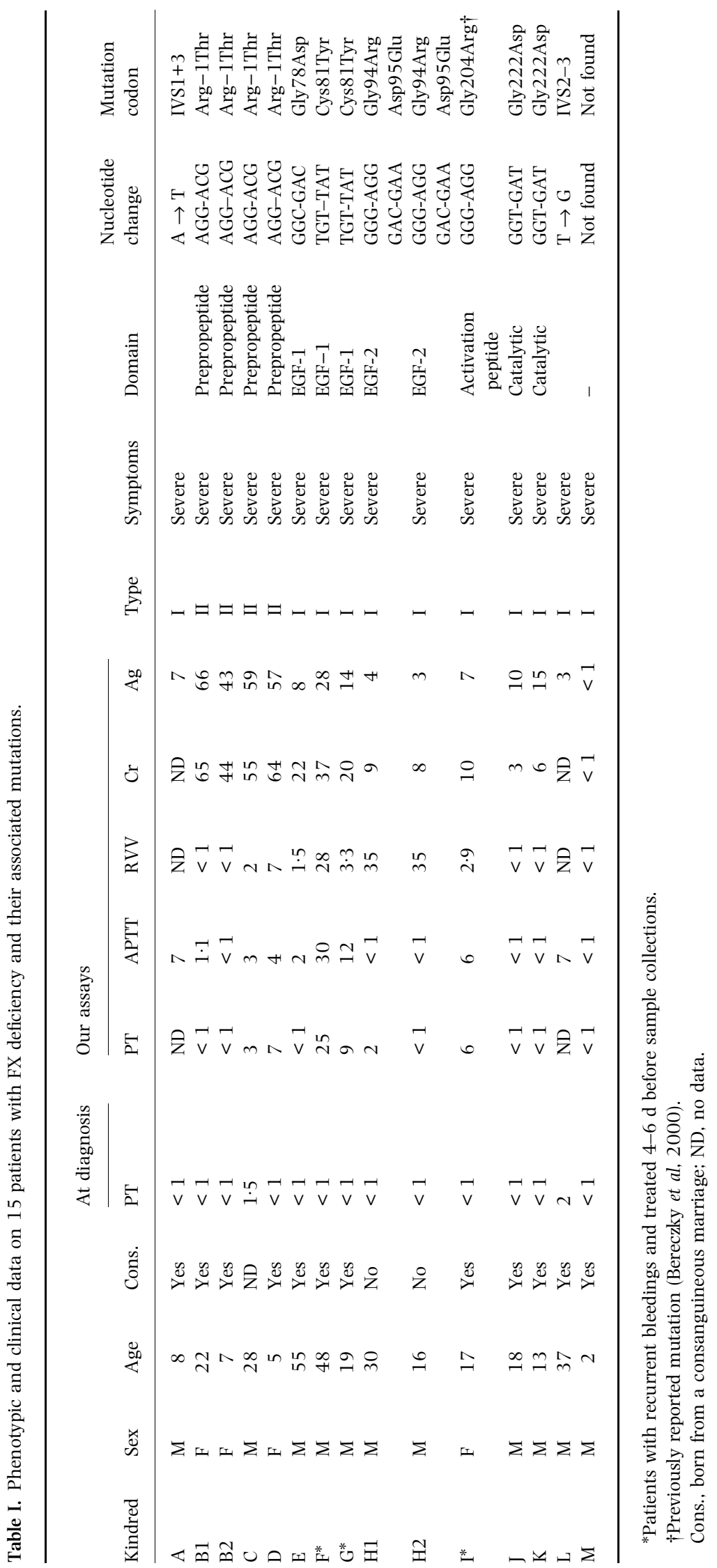


unstable and may cause intracellular degradation (Bereczky et al, 2000). None of the novel or previously reported mutations was found in the control Iranian population. In one patient with severe bleeding and type I deficiency $(\mathrm{M})$, no mutations were found. Patient $\mathrm{M}$ died following a CNS bleed at the age of 2 years.

\section{DISCUSSION}

The large number of homozygous candidate FX mutations presented here has significantly increased our knowledge on gene mutations associated with FX deficiency, the most severe of the rare recessively transmitted coagulation disorders. The identification of eight novel mutations in this study leads to a total of 47 known mutations in the FX gene (Fig 1).

In previous cases of FX deficiency, many patients were found to be heterozygous or doubly heterozygous for mutations in the FX gene (Cooper et al, 1997), making it difficult to assess the effect resulting from each allele. Here, we analysed the phenotype and genotype in a group of 15 patients with homozygous FX deficiency. The results of clotting assays in this study highlight the problems associated with screening FX deficiency. The PT, APTT and RVVbased assays showed similar sensitivities to both mild and severe reductions in FX. The chromogenic assay usually gave results similar to the FX:Ag level, but could not be used as a single assay because of the risk of an undiagnosed dysfunctional FX deficiency. In two patients from the same family (H1 and H2), a measurable coagulant activity by the RVV assay was found, which was in contrast with the undetectable FX:C activity measured by either a PT or APTT-based assay; the chromogenic or antigenic assays were also reduced (Simioni et al, 2001).

The genotype study by SSCP screening combined with direct sequencing of PCR products identified nine candidate mutations in the human FX gene in 13 kindred, of which eight were novel (Table I). In agreement with previously characterized mutants of FX, none of the mutations was predicted to lead to the production of a truncated protein or no protein at all, and this is consistent with the hypothesis that a complete absence of FX is fatal (Dewerchin et al, 2000). $\mathrm{FX}^{-1-}$ knockout mice present a lethal phenotype, which demonstrates the importance of FX function in embryonic and postnatal survival. Molecular graphics analyses based on crystal structures of FX were used to explain the effect of each of the novel mutations in comparison with the previously known ones.

\section{Arg-1Thr mutation}

The vitamin K-dependent $\gamma$-glutamyl carboxylase catalyses the post-translational modification of specific glutamic acid residues to $\gamma$-carboxyglutamic acid (Gla) residues in the Gla domain (Stanley et al, 1999a). This modification is essential for blood coagulation. In all known vitamin K-dependent proteins, the mechanism by which the carboxylase recognizes its substrate is believed to be through its initial binding to an essential conserved 18-residue propeptide. This propeptide is proteolytically removed before the formation of mature FX (Furie \& Furie, 1991). Mutations at the -1 position are not believed to affect carboxylation, but rather to disable propeptide cleavage and processing (Ware et al, 1989). The naturally occurring substitution at Arg-1Thr in four patients from three unrelated families is likely to inhibit this cleavage, and will produce only dysfunctional FX protein. Sequences alignment of the vitamin K-dependent protein propeptide demonstrated that Arg-1 is highly conserved (Stanley et al, 1999b). Naturally occurring missense mutations affecting the codon for the basic amino acid of the propeptidase recognition sequence of factor IX (Arg -1Ser) (Giannelli et al, 1994), protein C (Arg -1Cys/His/ Ser) (Miyata et al, 1995; Reitsma et al, 1995) and protein $S$ (Arg-1His) (Gandrille et al, 1995) all result in secretion of gene products with severely reduced functions.

\section{Four EGF mutations}

Out of the 39 previously known missense mutations shown in Fig 1, 23 occur in the serine protease domain, whereas only 13 occur in the Gla and EGF domains. Our present study has almost doubled the number of known mutations in the EGF domains of FX. The EGF-1 and EGF-2 domains are thought to be important not only in binding $\mathrm{Ca}^{2+}$ but also in maintaining the correct conformation of FXa. In the EGF-1 domain, the Gly78Asp and Cys81Tyr mutations correspond to exposed (Gly78) and buried (Cys81) residues at loop conformations that appear to be important for the correct folding of the EGF domain (Figs 1 and 2A). This is consistent with their identification as type I disorders. The replacement of the small Gly residue by a bulkier residue may prevent the EGF-1 domain from folding correctly. The loss of one out of the six Cys residues will prevent the correct formation of the three-disulphide bridges that are essential for the folding of the EGF-1 domain. In the EGF-2 domain, the simultaneous replacement of Gly94Arg and Asp95Glu occurs in a pocket containing two other acidic groups and three basic groups from the EGF-1, EGF-2 and catalytic domains (Fig 2B). The introduction of an extra charged residue in this region is likely to affect the correct folding of FX and, in turn, its secretion. This interpretation is in accordance with its assignment as a type I disorder. Of interest is that this double mutant shows only a mild reduction in coagulation activity by the RVV assay, and expression analysis will be required to verify the effect of this mutation.

\section{Catalytic domain mutation}

The catalytic activity of FX resides in the serine protease domain, in which the active site is formed from the triad of residues, His236, Asp282 and Ser379. The Gly222Asp mutation corresponds to the replacement of a tiny fully buried residue at the centre of a buried $\beta$-strand $\mathrm{C}$ by a larger charged residue adjacent to the catalytic triad (Fig 1). Although the introduction of this charged group may perturb the catalytic triad (Fig 2C), it is more likely that the insertion of a charged hydrophilic residue into the protein core will disrupt its correct folding. This interpretation is consistent with its type I assignment. 


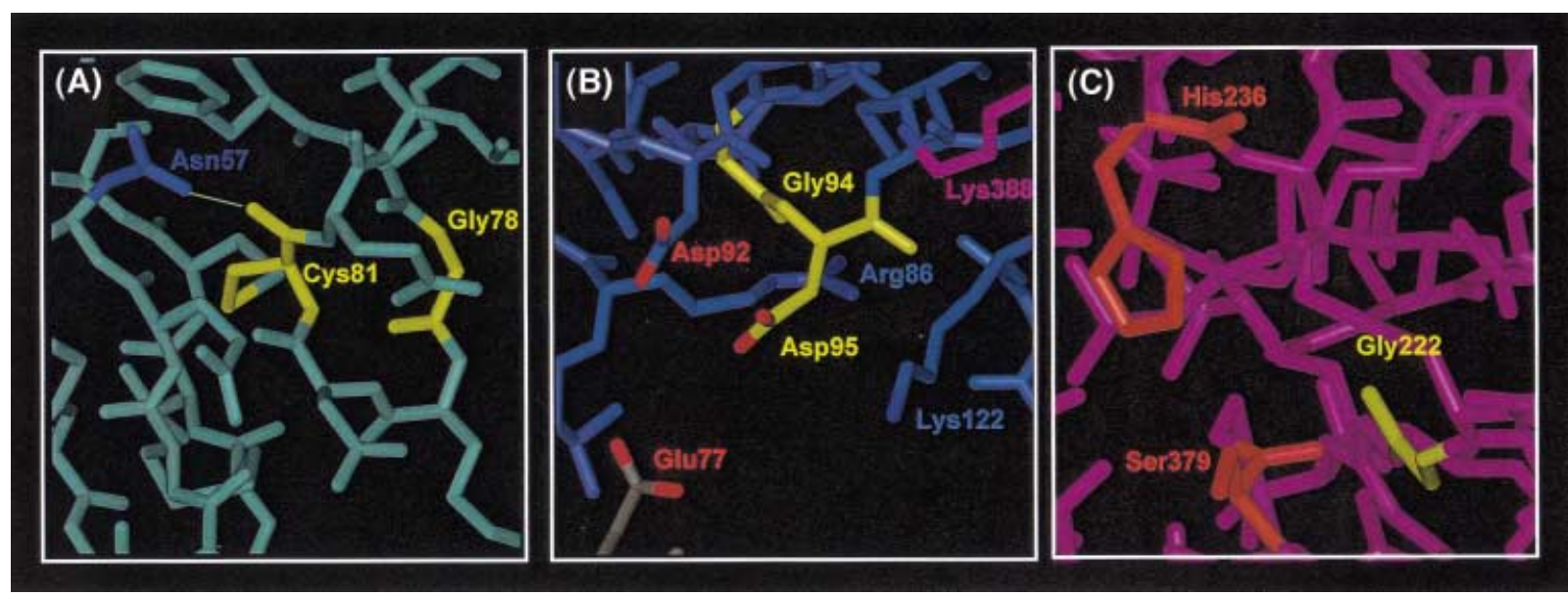

Fig 2. Structural role of the mutated amino acid residues that lead to type I FX disorders. (A) The EGF-1 domain is depicted with Cys81 and Gly 78 in yellow (Patients E, F and G). A hydrogen bond between the side-chain of Asn57 (blue) and the main-chain oxygen of Cys81 is shown in green. The side-chain of Gly78 is surface-exposed, whereas that of Cys81 is buried. (B) The EGF-2 domain is depicted, showing the double mutation residues at Gly94 and Asp95 in patients H1 and H2. The charged groups from the adjacent acidic residues Glu77 and Asp92 (red) and the adjacent basic residues Arg86, Lys122 (blue) and Lys388 (magenta) are shown. (C) In the catalytic domain, Gly222 (yellow: Patients J and $\mathrm{K}$ ) is shown in close proximity to His236 and Ser379 of the Asp-His-Ser catalytic triad (red). Asp282 is above the top of the figure as viewed, and is not shown for reason of clarity.

\section{Splice site mutations}

The use of the Berkely Drosophila Genome Project human database predicted that mutation IVS1+3 would probably result in the complete abolition of the donor-splicing site in mutant, whereas the IVS2-3 mutation would change the efficiency of the splicing from 1.00 (score for wild type FX) to 0.63 (score for mutant FX). These analyses accounted for the type I assignment of these two novel mutations.

Patients who are severely deficient in FX (with a reduced procoagulant activity and antigen level) are severe bleeders, and require much FX replacement therapy (Peyvandi et al, 1998). Therefore, prenatal diagnosis should be performed in pregnancies at risk in families with at least one member affected by this coagulation disorder, and knowledge of the underlying mutation is essential to achieve this goal.

\section{ACKNOWLEDGMENTS}

The financial support of the Katharine Dormandy Trust for Haemophilia and Allied Disorders, and the Angelo Bianchi Bonomi Foundation, is gratefully acknowledged. We thank Dr D. Owens, Dr R. Bader and Ms A. Riddell (Haemophilia Centre and Haemostasis Unit, Royal Free and University College Medical School, London and Italo Monzino Centre, Milan) for their assistance in coagulation assays. We also thank Dr M. Lak, Dr S. Zeinali (Haemophilia Centre and Pasteur Institute of Tehran) and the Iranian Haemophilia Society for their help and assistance in the identification and collection of samples.

\section{REFERENCES}

Bereczky, Z., Balogh, I., Ajzner, E., Kiss, C., Komaromi, I. \& Muszbek, L. (2000) Molecular genetic analysis of an inherited factor $\mathrm{X}$ deficiency. Haemostasis, 30, 35 (Abstract).
Brandstetter, H., Kuhne, A., Bode, W., Huber, R., von der Saal, W., Wirthensohn, K. \& Engh. R.A. (1996) X-ray structure of active site-inhibited clotting factor Xa. Implications for drug design and substrate recognition. Journal of Biological Chemistry, 271, 29988-29992.

Cooper, D.N., Millar, D.S., Wacey, A.I., Pemberton, S. \& Tuddenham, E.G.D. (1997) Inherited factor X deficiency: molecular genetics and pathophysiology. Thrombosis and Haemostasis, 78, 161-172.

Deam, S., Srinivasan, N., Westby, J., Horn, E.H. \& Dolan, G. (2001) FX Nottingham and FX Taunton: two novel mutations in factor $\mathrm{X}$ resulting in loss of functional activity and an interpretation using molecular modelling. Thrombosis and Haemostasis, 85, 265-269.

Dewerchin, M., Zhong, L., Moons, L., Carmeliet, P., Castellino, F.J., Collen, D. \& Rosen, E.D. (2000) Blood coagulation factor X deficiency causes partial embryonic lethality and fatal neonatal bleeding in mice. Thrombosis and Haemostasis, 83, 185-190.

Furie, B.M. \& Furie, B.C. (1988) The molecular basis of blood coagulation. Cell, 53, 505-518.

Furie, B. \& Furie, B.C. (1991) Molecular basis of gamma-carboxylation - Role of the propeptide in the vitamin K-dependent proteins. Annals of The New York Academy of Science, 614, 1-10.

Gandrille, S., Borgel, D., Eschwege-Gufflet, V., Aillaud, M., Dreyfus, M., Matheron, C., Gaussem, P., Abgrall, J.F., Jude, B., Sie, P., Toulon, P. \& Aiach, M. (1995) Identification of 15 different candidate casual point mutations and three polymorphisms in 19 patients with protein $S$ deficiency using a scanning method for the analysis of the protein S active gene. Blood, 85, 130-138.

Giannelli, F., Green, P.M., Sommer, S.S., Lillicrap, D.P., Ludwig, M., Schwaab, R., Reitsma, P.H., Goossens, M., Yoshioka, A. \& Brownlee, G.G. (1994) Haemophilia B: database of point mutations and short additions and deletions (5th edn). Nucleic Acid Research, 22, 3534-3546.

Iijima, K., Murakami, M., Kimura, O., Murakami, F., Shimomura, T. \& Ikawa, S. (2001) A dysfunctional Factor X (Factor X Kurayoshi) with a substitution of Arg 139 for Ser at the carboxyl-terminus of the light chain. Thrombosis Research, 101, 311-316. 
Kabsch, W. \& Sander, C. (1983) Dictionary of protein secondary structure: pattern recognition of hydrogen-bonded and geometrical features. Biopolymers, 22, 2577-2637.

Kamata, K., Kawamoto, H., Honma, T., Iwama, T. \& Kim, S.H. (1998) Structural basis for chemical inhibition of human blood coagulation factor Xa. Proceedings of the National Academy of Science of the United States of America, 95, 6630-6635.

Lak, M., Peyvandi, F. \& Mannucci, P.M. (2000) Prevalence of bleeding manifestations, hepatitis and alloantibodies to von Willebrand factor in 385 Iranian patients with type 3 von Willebrand Disease. British Journal of Haematology, 111, 1236-1239.

Lee, B. \& Richards, F.M. (1971) The interpretation of protein structures: estimation of static accessibility. Journal of Molecular Biology, 55, 379-400.

Leytus, S.P., Foster, D.C., Kurachi, K. \& Davie, E.W. (1986) Gene for human factor X: a blood coagulation factor whose gene organization is essentially identical with that of factor IX and protein C. Biochemistry, 25, 5098-5102.

Maignan, S., Guilloteau, J.P., Pouzieux, S., Choi-Sledeski, Y.M., Becker, M.R., Klein, S.I., Ewing, W.R., Pauls, H.W., Spada, A.P. \& Mikol, V. (2000) Crystal structures of human factor Xa complexed with potent inhibitors. Journal of Medicinal Chemistry, 43, 3226-3232.

Millar, D.S., Elliston, L., Deex, P., Krawczak, M., Wacey, A.I., Reynaud, J., Nieuwenhuis, H.K., Bolton-Maggs, P., Mannucci, P.M., Reverter, J.C., Cachia, P., Pasi, K.J., Layton, D.M. \& Cooper, D.N. (2000) Molecular analysis of genotype-phenotype relationship in factor X deficiency. Human Genetics, 106, 249-257.

Miyata, T., Zheng, Y.Z., Sakata, T. \& Kato, H. (1995) Protein C Osaka 10 with aberrant propeptide processing: loss of anticoagulant activity due to amino acid substitution in the protein $\mathrm{C}$ precursor. Thrombosis and Haemostasis, 74, 1003-1008.

Miyata, T., Fischer, F., Umeyama, H., Appert, A., Jambou, D. \& Kato, H. (1998a) Factor X Nice I and II: two novel missense mutations (Met-40Val and Pro304Ser) in patient with coagulation factor X deficiency. Thrombosis and Haemostasis, 80, 709-710.

Miyata, T., Kojima, T., Suzuki, K., Umeyama, H., Yamazaki, T., Kamiya, T., Toyoda, H. \& Kato, H. (1998b) Factor X Nagoya 1 and Nagoya 2: a CRM-factor X deficiency and a dysfunctional CRM+ factor $\mathrm{X}$ deficiency characterized by substitution of Arg 306 by Cys and of Gly366 by Ser, respectively. Thrombosis and Haemostasis, 79, 486-490.

Nobauer-Huhmann, I.M., Holler, W., Krinninger, B., Turecek, P.L., Richter, G., Scharrer, I., Forberg, E. \& Watzke, H.H. (1998) Factor $\mathrm{X}$ Frankfurt I: molecular and functional characterization of a hereditary factor X deficiency (Gla+25 to Lys). Blood Coagulation and Fibrinolysis, 9, 143-152.

Orita, M., Suzuki, Y., Sekiya, T. \& Hayashi, K. (1989) Rapid and sensitive detection of point mutations and DNA polymorphisms using polymerase chain reaction. Genomics, 5, 874-879.
Padmanabhan, K., Padmanabhan, K.P., Tulinsky, A., Park, C.H., Bode, W., Huber, R., Blankenship, D.T., Cardin, A.D. \& Kisiel, W. (1993) Structure of human des(1-45) factor Xa at $2.2 \AA$ resolution. Journal of Molecular Biology, 232, 947-966.

Perry, D.J. (1997) Factor X and its deficiency states. Haemophilia, 3, 159-172.

Peyvandi, F. \& Mannucci, P.M. (1999) Rare coagulation disorders. Thrombosis and Haemostasis, 82, 1207-1214.

Peyvandi, F., Mannucci, P.M., Lak, M., Abdoullahi, M., Zeinali, S., Sharifian, R. \& Perry, D. (1998) Congenital factor X deficiency: spectrum of bleeding symptoms in 32 Iranian patients. British Journal of Hematology, 102, 626-628.

Reitsma, P.H., Bernardi, F., Doig, R.G., Gandrille, S., Greengard, J.S., Ireland, H., Krawczak, M., Lind, B., Long, G.L., Poort, S.R., Saito, H., Sala, N., Witt, I. \& Cooper, D.N. (1995) Protein C deficiency: a database of mutations. Thrombosis and Haemostasis, 73, $876-889$.

Sali, A. \& Blundell, T.L. (1990) The definition of topological equivalence in homologous and analogous structures: a procedure involving a comparison of local properties and relationships. Journal of Molecular Biology, 212, 403-428.

Simioni, P., Vianello, F., Kalafatis, M., Barzon, L., Ladogana, S., Paolucci, P., Carotenuto, M., Dal Bello, F., Palu, G. \& Girolami, A. (2001) A dysfunctional Factor X (Factor X San Giovanni Rotondo) present at homozygous and double heterozygous level: identification of a novel microdeletion (delC556) and missense mutation (Lys (408) $\rightarrow$ Asn) in the Factor X gene. A study of an Italian family. Thrombosis Research, 101, 219-230.

Stanley, T.B., Jin, D., Lin, P.J. \& Stafford, D.W. (1999a) The propeptides of the vitamin K-dependent proteins possess different affinities for the vitamin K-dependent carboxylase. Journal of Biological Chemistry, 274, 16940-16944.

Stanley, T.B., Humphries, J. \& High, K.A. \& Stafford, D.W. (1999b) Amino acids responsible for reduced affinities of vitamin $\mathrm{K}$-dependent propeptides for the carboxylase. Biochemistry, 38, 15681-15687.

Tuddenham, E.G.D. \& Cooper, D.N. (1994) The Molecular Genetics of Haemostasis and its Inherited Disorders. Oxford University Press, Oxford.

Ware, J., Diuguid, D.L., Liebman, H.A., Rabiet, M.J., Kasper, C.K., Furie, B.C., Furie, B. \& Stafford, D.W. (1989) Factor IX San Dimas. Substitution of glutamine for Arg-4 in the propeptide leads to incomplete gamma-carboxylation and altered phospholipid binding properties. Journal of Biological Chemistry, 264, 11401-11406.

Zama, T., Murata, M., Watanabe, R., Yokoyama, K., Moriki, T., Ambo, H., Murukami, H., Kikuchi, M. \& Ikeda, Y. (1999) A family with hereditary factor $\mathrm{X}$ deficiency with a point mutation Gla32 to Gln in the Gla domain (factor X Tokyo). British Journal of Haematology, 106, 809-811. 